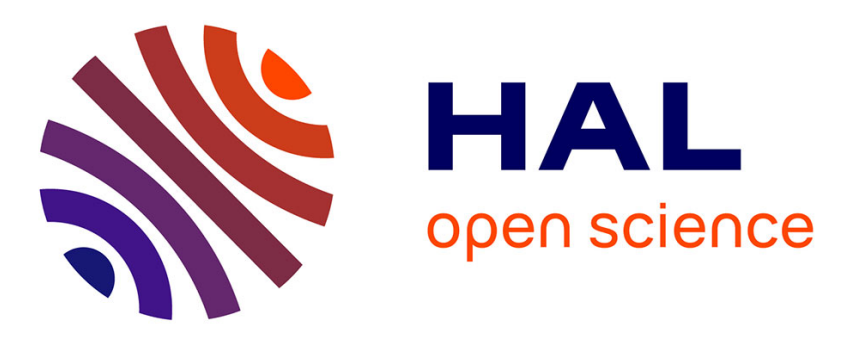

\title{
High resolution frequency standard dissemination via optical fiber metropolitan network
}

\author{
F Narbonneau, M Lours, S Bize, A Clairon, G Santarelli, O Lopez, Ch \\ Daussy, Anne Amy-Klein, Ch Chardonnet
}

\section{- To cite this version:}

F Narbonneau, M Lours, S Bize, A Clairon, G Santarelli, et al.. High resolution frequency standard dissemination via optical fiber metropolitan network. Review of Scientific Instruments, 2006, 10.1063/1.2205155 . hal-03151495

\section{HAL Id: hal-03151495 \\ https://hal.science/hal-03151495}

Submitted on 2 Mar 2021

HAL is a multi-disciplinary open access archive for the deposit and dissemination of scientific research documents, whether they are published or not. The documents may come from teaching and research institutions in France or abroad, or from public or private research centers.
L'archive ouverte pluridisciplinaire HAL, est destinée au dépôt et à la diffusion de documents scientifiques de niveau recherche, publiés ou non, émanant des établissements d'enseignement et de recherche français ou étrangers, des laboratoires publics ou privés. 


\title{
High Resolution Frequency Standard Dissemination via Optical Fibre Metropolitan Network
}

\author{
F. Narbonneau, M. Lours, S. Bize, A. Clairon, and G. Santarelli \\ LNE-SYRTE, Observatoire de Paris, 61 Avenue de l'Observatoire, 75014 Paris, France \\ O. Lopez, Ch. Daussy, A. Amy-Klein, and Ch. Chardonnet \\ Laboratoire de Physique des Lasers, Université Paris XIII, Villetaneuse, France
}

\begin{abstract}
We present in this paper results on a new dissemination system of ultra-stable reference signal at $100 \mathrm{MHz}$ on a standard fibre network. The $100 \mathrm{MHz}$ signal is simply transferred by amplitude modulation of an optical carrier. Two different approaches for compensating the noise introduced by the link have been implemented. The limits of the two systems are analyzed and several solution suggested in order to improve the frequency stability and to further extend the distribution distance. Nevertheless, our system is a good tool for the best cold atom fountains comparison between laboratories, up to $100 \mathrm{~km}$, with a relative frequency resolution of $10^{-14}$ at one second integration time and $10^{-17}$ for one day of measurement. The distribution system may be upgraded to fulfill the stringent distribution requirements for the future optical clocks.
\end{abstract}

\section{INTRODUCTION}

Ultra-stable frequency and time sources play an important role in many modern Time and Frequency metrology and fundamental physics applications (clock evaluation, relativity tests, fundamental constants test ...)(e.g. [1], 2], 3], [4]). In the field of particles physics, modern large linear accelerators require RF distribution system with minimal phase drifts and errors for the neutrons and positrons generation [5]. In radio-astronomy, e.g. in the case of the ALMA (Atacama Large Millimetric Array) project or for VLBI (Very Long Baseline Interferometry), the combination of high frequency and long baselines of the interferometer needs the distribution of a local oscillator with low phase noise and low phase drift through the array [6], 7]. For the Deep Space Network (DSN), the Jet Propulsion Laboratory (JPL) has developed a fibre link to distribute reference signals from an H-Maser to synchronize each antenna of the DSN [8], [9].

Modern cold atoms frequency standards in the microwave domain have already demonstrated an accuracy in the $10^{-15}$ range with the potential to reach the $10^{-16}$ level or better. Frequency stabilities, defined by the Allan standard Deviation (ADEV), are commonly of $10^{-13} \tau^{-\frac{1}{2}}$ for such standards and a few $10^{-14} \tau^{-\frac{1}{2}}$ have been demonstrated using more advanced techniques [10]. Cold atom optical clocks have the potential to reach the $10^{-17}$ accuracy level [1], [12], 13], 14]. The emergence of modern microwaveto-optical synthesizers based on mode-locked femtosecond lasers allows high resolution comparisons between microwave and optical clocks 15], 16], 17]. Clocks comparisons are currently performed by satellite, as for example GPS or TWSTFT (Two-Way Satellite Time and Frequency Transfer. Measurements are limited by the transmission system to about $10^{-15}$ at one day averaging time [18. Theses methods are thus insufficient for measuring the ultimate performance of a microwave

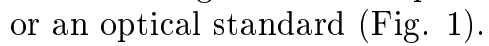

Upgrades of the orbital equipments are expectable to improve the current performance, but are quite

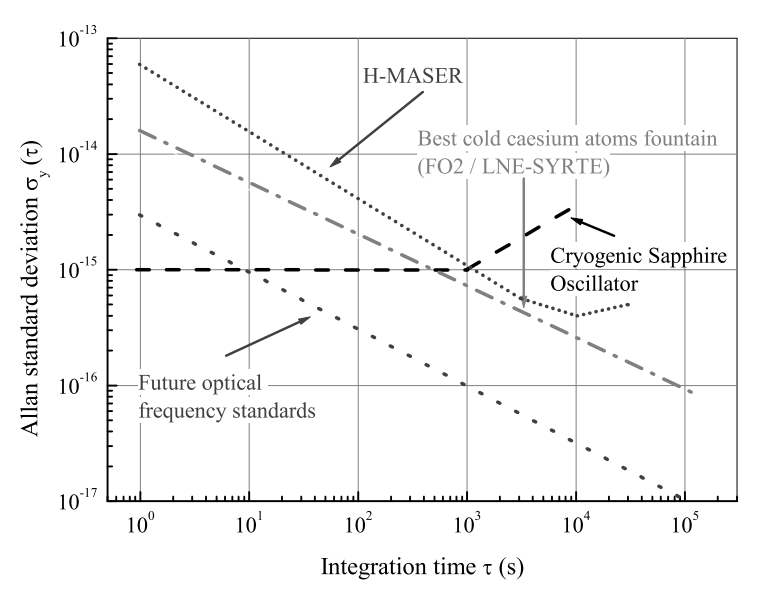

Figure 1: Allan deviation of some frequency standards

complex and expensive. Moreover, the two previous systems deliver only a synchronization signal not allowing direct short-term stability comparisons. Then for much of applications a reference signal is needed. Hence, the opportunity to compare microwave and optical clocks by the development of a new type of a ground frequency dissemination by optical fibre seems appropriate, even when the laboratories are separated by $100 \mathrm{~km}$ [19], [20], 21]. One can indeed take advantage of both the low attenuation and low dispersion in the fibre, which allow reaching long distance frequency transfer by maintaining a good signal-to-noise ratio (SNR).

Moreover the access to an ultra-stable frequency reference for a large number of laboratories open the way to perform new experiments in fundamental physics. The development and operation of a state-of-the-art frequency standard remain a strong limitation and can be overcome by a fibre distribution system connecting Time and Frequency Metrology laboratories to users. The simplest way to develop a fibre distribution is to use the redundancy of the telecom network. In this pa- 
per, we present the transfer of high frequency stability signal at $100 \mathrm{MHz}$, by using the existing telecommunication fibre network, over a few tens kilometers, with compensation of the phase noise introduced by the link.

\section{PRINCIPLE AND OBJECTIVE}

The goal of the dissemination is the distribution of a reference signal at a frequency of $100 \mathrm{MHz}$, synthesized from a frequency standard, by amplitude modulation of an optical carrier, without degradation of the phase noise of the distributed signal. The reference signal modulates the bias current of a DFB laser diode, at $1.55 \mu \mathrm{m}$, which is transmitted through a fibre optical link to users. At the link extremity, a photodiode detects the amplitude modulation and converts the optical signal to a radio-frequency signal oscillating at the reference frequency and phase coherent with the microwave reference source.

The high stability and low phase noise of the transferred signal are degraded by the residual phase noise of the optical link and by the attenuation in the fibre. We operate in urban environment by using the existing telecom network. Thus, fibre layout and installation aspects are not ideal and the stability of the optical link can be affected by environmental effects. Optical length of the fibre is modified by mechanical stresses and temperature fluctuations. The first one affects phase noise and short-term frequency stability performances of the transmitted signal. The second effect, is a slowly changing phenomenon and has an impact on the long-term stability.

These instabilities have been studied on two optical links using the dense France Telecom network and connecting LNE-SYRTE to Laboratoire de Physique des Lasers (LPL) (about $43 \mathrm{~km}$ ), and LNE-SYRTE with Laboratoire Kastler Brossel (LKB - University Paris VI) (about $3 \mathrm{~km}$ ).

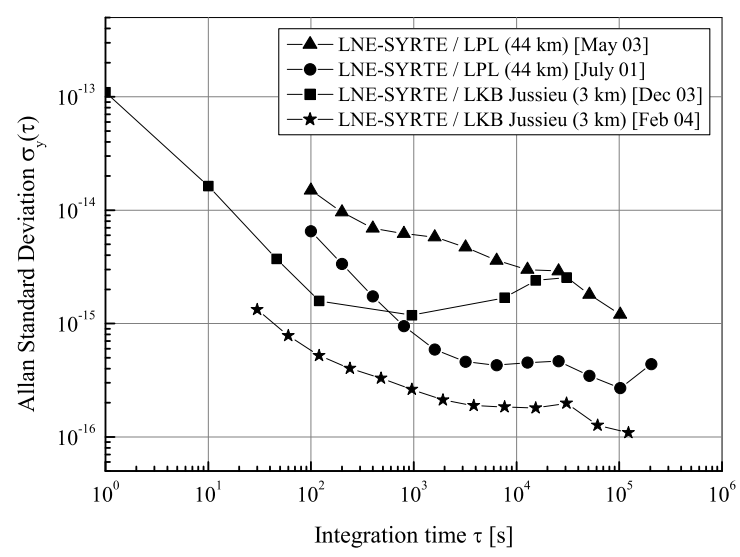

Figure 2: Frequency stability measurements of the LNESYRTE/LPL and LNE-SYRTE/LKB optical links

Measurements, realized at different periods, are pre- sented in figure 2 and show non-stationary effects de 2 pending on the activities around the link. Periodic effects as daily temperature variations appears as a bump at the half period, on the ADEV. The frequency instabilities related to a sinusoidal temperature perturbations can be calculated from the equation (1):

$$
\sigma_{y}(\tau)=\Delta T \times \frac{T C D \times n \times L}{c} \times \frac{\sin ^{2}\left(\pi \tau \nu_{0}\right)}{\tau}
$$

with $\Delta T$ the amplitude of the temperature fluctuation $\left[{ }^{\circ} \mathrm{C}\right], T C D$ the thermal coefficient of delay $\left[\mathrm{ppm} /{ }^{\circ} \mathrm{C}\right]$ of the optical fibre (typically $7 \mathrm{ppm} /{ }^{\circ} \mathrm{C}$ for standard telecom SMF28 fibre), $n$ the fibre core index, $L$ the optical link length $[\mathrm{km}], c$ the light velocity in vacuum $\left[3 \times 10^{8} \mathrm{~m} / \mathrm{s}\right], \nu_{0}$ the perturbation frequency [Hz], and $\tau$ the averaging time [s]. For example, if we consider a sinusoidal perturbation of $0.2^{\circ} \mathrm{C}$ with a period of $1000 \mathrm{~s}$ due to air conditioning and acting on a section of 50 meters of the optical link, the ADEV of the link could be limited to about $7 \times 10^{-16}$ at $500 \mathrm{~s}$ integration time. In the same way, a daily $0.5^{\circ} \mathrm{C}$ temperature variation on 43 kilometers of optical fibre is converted into an instability of the order of $1.3 \times 10^{-14}$ at $43200 \mathrm{~s}$ averaging time.

Consequently, the distribution system needs an active control loop to compensate for these phase variations induced on the signal transmitted through the link related to the environment (mechanical vibrations, temperature fluctuations ...).

The objective of the dissemination being clock comparisons or delivery of a reference signal coming from an H-Maser or a Cryogenic Sapphire Oscillator (CSO), the compensation set-up must introduce a phase noise lower than the reference signal. In this perspective we have to develop a system which delivers a reference signal at $100 \mathrm{MHz}$, showing a relative frequency stability $\sigma_{y}(\tau) \leq 2.10^{-14}[\tau=1 \mathrm{~s}]\left(<10^{-16} @ 1 \mathrm{~d}\right)$, that implies a residual flicker phase noise of $-120 \mathrm{dBrad}^{2} / \mathrm{Hz}$ at 1 $\mathrm{Hz}$ and a white phase noise floor with a level of -140 $\mathrm{dBrad}^{2} / \mathrm{Hz}$.

\section{ACTIVE PHASE FLUCTUATIONS COMPENSATION SYSTEM}

\section{A. Presentation}

The principle of the phase fluctuations compensation, is displayed in figure 3. At the link extremity, the detected signal can not be directly compared to the reference signal and thus the correction of the phase perturbations can be only carried out at the link emission. A two-way distribution, using the same optical fibre link, allows determination of the phase perturbation accumulated along a full round trip with the hypothesis that the forward and the backward signals are corrupted by the same perturbation. The compensation rests then on the measurement of the phase of the signal after one round trip to apply a correction on the emitted signal. 


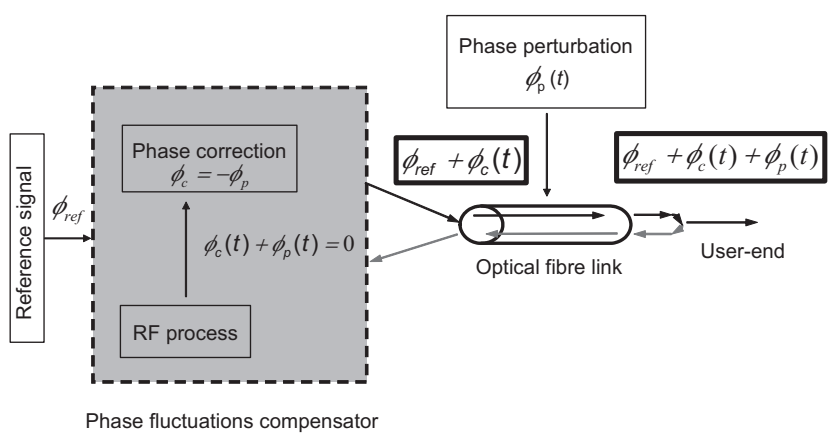

Figure 3: Schematic of the phase fluctuations compensation

The reference signal at the frequency $\mathrm{f}_{\mathrm{ref}}=\omega_{\mathrm{ref}} / 2 \pi$ is used for modulating a laser diode. The amplitude modulated signal is then corrected by a phase term $\phi_{c}$. This correction term is provided either by phase shifting the RF modulating signal or by modifying the propagation delay in the fibre. At the user-end, the signal corrupted by the environmental perturbations is detected:

$$
V_{\mathrm{RF} \text { detected }}(t) \propto \sin \left(\omega_{\text {ref }} t+\phi_{\text {ref }}+\phi_{c}+\phi_{p}\right)
$$

This signal is split in two signals: one part for the user applications and the other to be re-injected via an optical circulator in the same optical fibre. After one round-trip, the signal, twice corrupted by the term $\phi_{p}$ is detected. A RF process allows generation of an error signal, applied to the phase corrector. Two different laser sources, operating at slightly different wavelengths, are used for generating the forward and the backward optical signals and optical add/drop functions are realized with optical circulators.

Different approaches of phase compensation have been studied and are described here.

\section{B. Electronic phase fluctuations compensator}

In the case of an electronic phase fluctuations compensator (cf fig. 4), the correction is performed by acting on the phase of the injected signal in the optical link, that we call $\phi_{\text {input }}$.

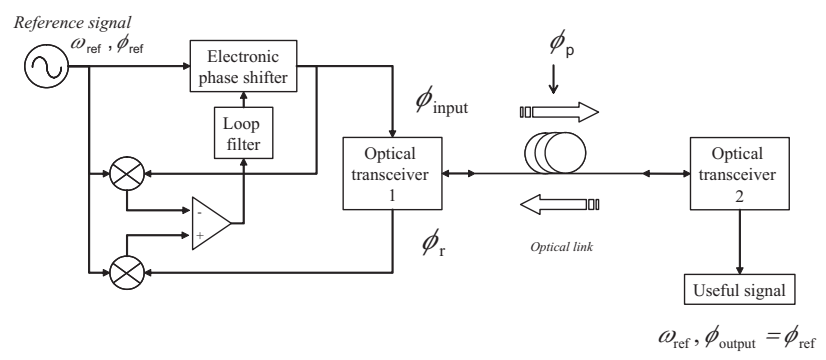

Figure 4: Simplified schematic of the phase conjugator

We define by $\phi_{r}$ the phase of the round-trip signal, and $\phi_{\text {output }}$ the phase of the detected signal at the user- end, equal to:

$$
\phi_{\text {output }}(t)=\phi_{\text {input }}(t-\tau)+\int_{t-\tau}^{t} \phi_{p}(\xi) d \xi
$$

where $\tau$ is the propagation delay in the optical fibre link and $\phi_{p}(\xi)$ is the distributed phase perturbation along the fibre. The main effect of the delay $\tau$ is to limit the loop bandwidth. In the following discussion, we neglect the influence of the delay.

The output signal must be phase coherent with the reference source of frequency $\omega_{\text {ref }}$ and on average of phase $\phi_{\text {ref }}$, and thus the correction applied to the emitted signal must be equal to the opposite of the phase perturbation $\phi_{p}$. Consequently, on average (or for time much longer than $\tau$ ) the phase of the input signal, $\phi_{\text {input }}$ is:

$$
\phi_{\text {input }}=\phi_{\text {ref }}-\phi_{p}
$$

Then, the phase of the round-trip signal becomes:

$$
\begin{aligned}
\phi_{r} & =\phi_{\text {input }}+2 \times \phi_{p} \\
& =\phi_{\text {ref }}+\phi_{p}
\end{aligned}
$$

The phase coherence of the output signal is hence imposed by maintaining a conjugation relationship between the input and the round trip signal of the optical link:

$$
\left(\phi_{\text {input }}-\phi_{\text {ref }}\right)=-\left(\phi_{r}-\phi_{\text {ref }}\right)
$$

A simplified scheme of the phase conjugator is shown in figure 4 The correction is performed with a phase shifter in series with the reference signal, which is used as the input signal. The reference signal is power divided to drive two phase detectors. Phase detection between the reference signal, the input corrected signal and the round-trip signal, allow generation of two baseband signals, connected to the inputs of a low noise differential amplifier. The output of this amplifier is used for driving a loop filter, controlling the electronic phase shifter until the phase conjugation, and thus a zero level at the amplifier output is reached. Although the simplicity of operation, this system suffers from various drawbacks. First, the phase correction is limited by the dynamic of the phase shifter. Electronic phase shifters have a typical dynamic of 180 degrees with a non linear response, inducing variable insertion losses. Moreover the phase shifter can present a phase noise excess, compared to the other components of the phase conjugator. Secondly, phase detectors are quite sensitive to the driving levels and it is difficult to ensure the same sensitivity for the two detectors of figure 4. The practical realization leads to a very poor effective system of the phase perturbations cancellation. A new scheme, regarding the previous considerations and introduced by the JPL [22] is shown in figure 5]

Two symmetrical signals are produced by frequency shift $\left(f_{\text {shift }}\right)$ of the reference signal $\left(f^{ \pm}=f_{\text {ref }} \pm f_{\text {shift }}\right)$. 


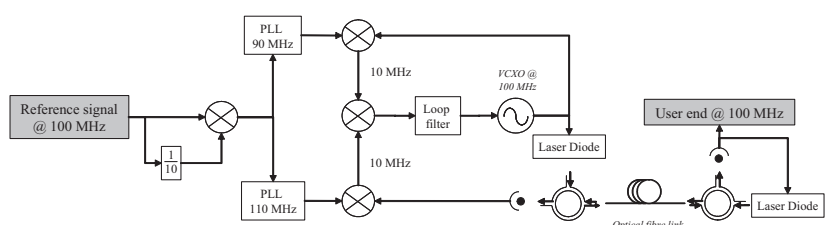

Figure 5: Block diagram of the full electronic compensation system

This scheme allows replacement of the double phase measurements (Fig. 4) by a much more accurate double frequency mixing and a single phase measurement. The dynamic and the linearity of the phase correction is improved by using a voltage controlled quartz oscillator (VCXO), as a phase shifter, delivering a signal at the reference frequency with a stable amplitude. The VCXO presents thus the advantage to correct all phase perturbation in the correction bandwidth of the phase compensator, which is limited by the round-trip propagation delay in the optical link (about $0.3 \mathrm{~ms}$ in the case of the 43-km LNE-SYRTE to LPL optical link).

The $100 \mathrm{MHz}$ output signal of the VCXO modulates the bias current of the DFB laser diode. The optical signal is launched in the optical fibre link to the user. At the user end, a simple system allows detection and regeneration of the backward signal. The detected signal after a one-way distribution is proportional to:

$$
V_{\text {User end }}(t) \propto \sin \left(\omega_{\text {osc }} \times t+\phi_{\text {osc }}+\phi_{p}\right)
$$

The backward optical signal is submitted to the same phase perturbation and after one complete round-trip, the detected signal has the following form:

$$
V_{\text {round trip }}(t) \propto \sin \left(\omega_{\text {osc }} \times t+\phi_{\text {osc }}+2 \times \phi_{p}\right)
$$

The servo loop forces the VCXO at $100 \mathrm{MHz}$ both to be phase coherent with the reference source and to compensate for the phase perturbation. For obtaining the phase conjugation, two signals separated by $10 \mathrm{MHz}$ around the reference frequency (one at $90 \mathrm{MHz}$ and the other at $110 \mathrm{MHz}$ ) are produced by frequency mixing between the reference signal and itself frequency divided by ten. Two different systems, based on PLL (Phase Lock Loop) are used for filtering each signal issue from the previous frequency mixing. The signal, from the "down conversion", at $90 \mathrm{MHz}$, is mixed with the modulating signal, delivered by the VCXO, to obtain a signal at $10 \mathrm{MHz}$ :

$V_{1}(t) \propto \sin \left(\left(\omega_{\mathrm{osc}}-2 \pi \times 90 \mathrm{MHz}\right) \times t+\phi_{\mathrm{osc}}-\frac{9}{10} \phi_{\mathrm{ref}}\right)$

In parallel, the signal at $110 \mathrm{MHz}$ is mixed with the round-trip signal, producing another signal at $10 \mathrm{MHz}$ :

$$
V_{2}(t) \propto \sin \left(\left(2 \pi \times 110 \mathrm{MHz}-\omega_{\mathrm{osc}}\right) \times t+\frac{11}{10} \phi_{\mathrm{ref}}-\phi_{\mathrm{osc}}-2 \phi_{p}\right)
$$

The phase comparison at $10 \mathrm{MHz}$ allows generation of a base-band signal, containing the three phase terms:

$$
V_{\text {error }}(t) \propto \phi_{\text {osc }}+\phi_{p}-\phi_{\text {ref }}
$$

which is cancelled in normal operation. The phase of the VCXO is then:

$$
\phi_{\mathrm{osc}}=\phi_{\mathrm{ref}}-\phi_{p}
$$

By this process, the stability and the accuracy of the reference source is transmitted to the user end in the system bandwidth.

The capacity of the phase compensator to reject phase perturbations in the control bandwidth is defined by the rejection factor, equal to the ratio between the phase variations in open and in closed loop. The performance of the distribution system depend both of the intrinsic system phase noise and of the rejection factor.

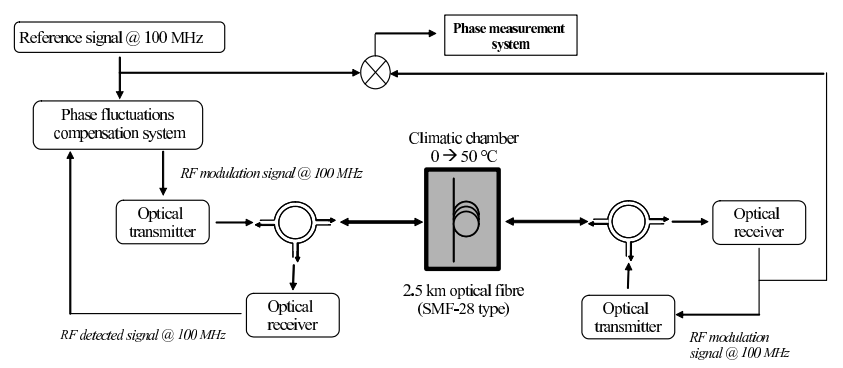

Figure 6: Block diagram of the compensation system test bench

Figure 6] displays the set-up used for the characterization of the phase conjugator. Simulation of phase perturbations are realized by periodically heating a $2.5-\mathrm{km}$ fibre spool with an amplitude of $4^{\circ} \mathrm{C}$ and a period of about $4000 \mathrm{~s}$. This perturbation induces a phase modulation of the order of $200 \mathrm{mrad}$ on the $100-\mathrm{MHz}$ transmitted signal. In operation, when the phase conjugator is activated, the residual phase modulation measured at the link output is reduced to $0.4 \mathrm{mrad}$ (cf. figure 7), that implies a rejection factor of the phase perturbations along the link of about 500 $(53 \mathrm{~dB})$.

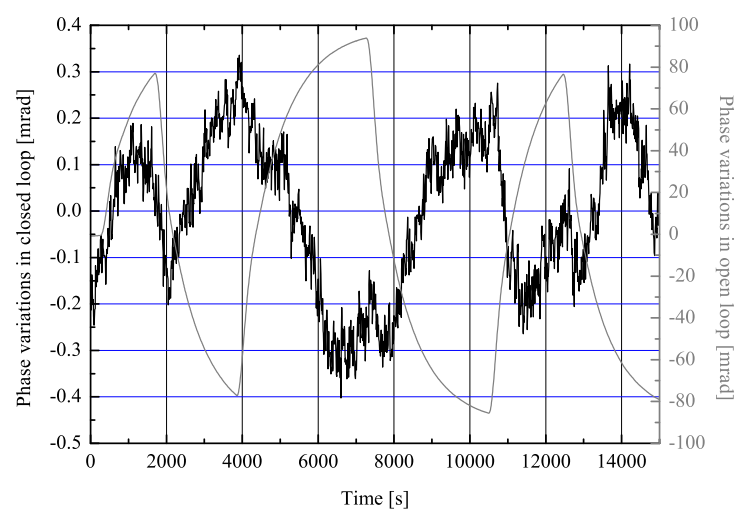

Figure 7: Phase shift induced by temperature modulation of the transmitted signal, in open and closed loop at 100 $\mathrm{MHz}$ 
Moreover, the complete distribution system including the phase conjugator shows a flicker phase noise with a level of $-123 \mathrm{dBrad}^{2}$ at $1 \mathrm{~Hz}$ and a white noise floor below $-140 \mathrm{dBrad}^{2} / \mathrm{Hz}$ (Fig. 8). This ensures the possibility to transfer metrological signal with a frequency stability $\sigma_{y}(\tau)$ below than $1 \times 10^{-14}$ at 1 second averaging time.

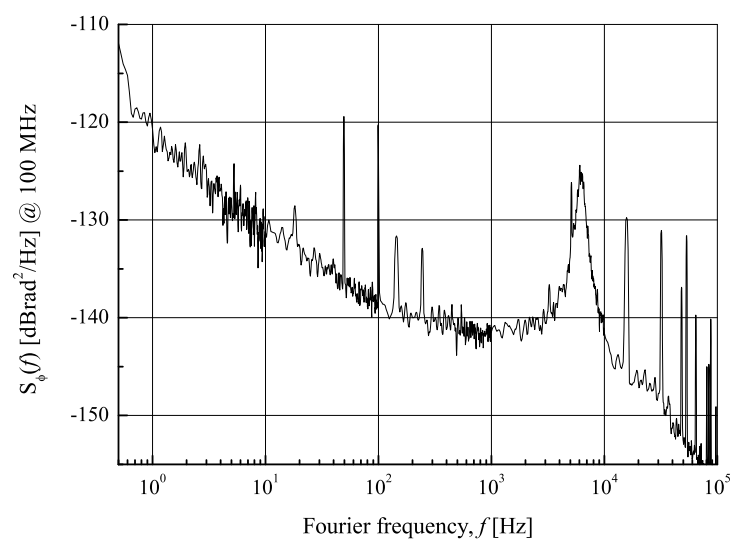

Figure 8: Phase noise spectral density of the full electronic compensation system (The phase noise bump comes from a gain excess of the loop)

This system was implemented at SYRTE.

\section{Optical compensation system}

An optoelectronic compensation system has also been developed and implemented at LPL, operating in a slightly different way. The phase correction is applied both on the emitted and on the backward signal by directly acting on a section of optical fibre, placed in series with the optical link. The phase correction is then performed by modifying the optical propagation delay (and thus the optical path) of the optical signal in the fibre link. The principle of the optoelectronic phase compensation is presented in figure 9.

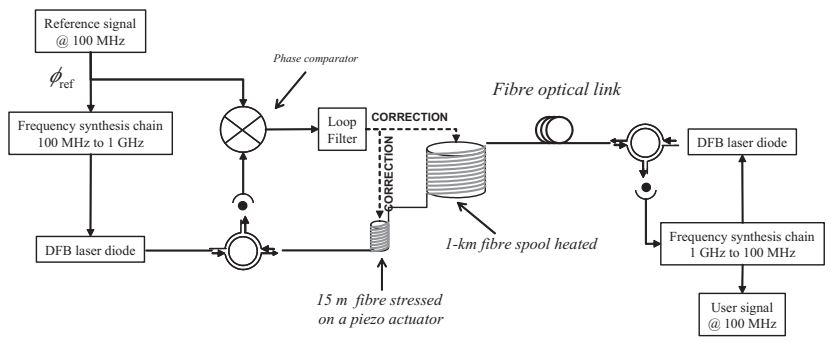

Figure 9: Schematic of the optoelectronic system for phase fluctuations compensation

For increasing isolation between the two ways of the distribution system, two different modulation frequencies are used: $1 \mathrm{GHz}$ for the emission and $100 \mathrm{MHz}$ for the return way. Consequently, a $1-\mathrm{GHz}$ signal must be generated from the reference $100-\mathrm{MHz}$ signal by 5 frequency synthesizer. The optical signal is distributed in the fibre link through the optical compensator and thus corrected. At the user-end, the detected signal has the following form:

$$
V_{\text {user }} \propto \sin \left(2 \pi \times 1 \mathrm{GHz} \times t+10 \times \phi_{\text {ref }}+\phi_{\text {correction }}^{\prime}+\phi_{p}^{\prime}\right)
$$

where $\phi_{\text {correction }}^{\prime}$ and $\phi_{p}^{\prime}$ are respectively the correction term and the perturbation applied to the transmitted signal at $1 \mathrm{GHz}$.

A second frequency synthesis chain allows delivering a signal at $100 \mathrm{MHz}$ from the $1-\mathrm{GHz}$ detected signal. This signal is used for modulating a second laser diode to produce the backward optical signal. This signal is corrupted by the same perturbation and also corrected. After one round trip, we detect a $100 \mathrm{MHz}$ signal proportional to:

$V_{\text {round trip }} \propto \sin \left(2 \pi \times 100 \mathrm{MHz} \times t+\phi_{\text {ref }}+2 \times\left(\phi_{\text {correction }}+\phi_{p}\right)\right)$

with:

$$
\begin{aligned}
\phi_{\text {correction }} & =\frac{\phi_{\text {correction }}^{\prime} \text { and }}{10} \\
\phi_{p} & =\frac{\phi_{p}^{\prime}}{10}
\end{aligned}
$$

The phase comparison between the reference signal at $100 \mathrm{MHz}$ and the round-trip signal leads to generation of a baseband error signal:

$$
V_{\text {error }} \propto \phi_{\text {correction }}+\phi_{p}
$$

which is applied to a loop filter to drive the optical phase corrector. This corrector is composed of two subsystems. Fast and small phase fluctuations (mechanical vibrations, fast temperature variations induced by air conditioning eg.) are corrected by changing the length of a portion of optical fibre with a piezo-electric actuator. A 15-meter optical fibre is wrapped around a 5 -cm diameter PZT of about $10 \mu \mathrm{m}$ variation under $1 \mathrm{kV}$ voltage, and is mechanically stretched, allowing correction up to about $15 \mathrm{ps}$ or $10 \mathrm{mrad}$ at $100 \mathrm{MHz}$, in a bandwidth of a few hundreds $\mathrm{Hz}$.

Slow and large perturbation are compensated by heating a $1-\mathrm{km}$ fibre spool introduced along the link $(\simeq 40$ $\mathrm{ps} /{ }^{\circ} \mathrm{C}$ or $25 \mathrm{mrad} /{ }^{\circ} \mathrm{C}$ at $100 \mathrm{MHz}$ ).

For the laboratory tests, a fibre stretcher and an heating system are placed along a laboratory link of a few kilometers to generate fast and slow phase perturbations. By this way, a temperature step of $10{ }^{\circ} \mathrm{C}$ is realized on a 1-km fibre spool, and the phase shift induced on the detected signal and measured in open and closed loop is reported in Fig. [10. Rejection factor of about 750 for slow phase perturbations is shown.

In contrast, only 20-25 dB attenuation (Fig. [11) are observed on intentionally produced small and fast perturbations. The gain of the correction is limited by parasitic phase shifts generated by Polarization Dependent Losses (PDL) under mechanical stress of the fibre. The mechanical stress affects the geometry of the fibre which becomes birefringent. Thus the polarization of the transmitted beam changes and leads to 


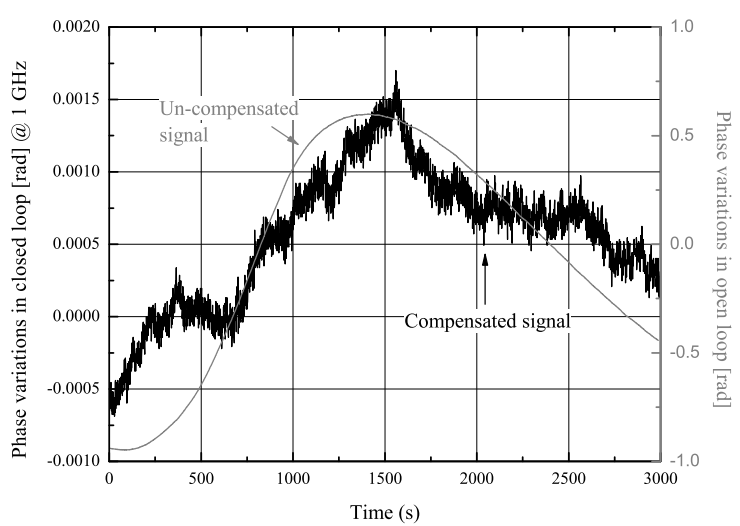

Figure 10: Phase variations measurement, induced by a 10 ${ }^{\circ} \mathrm{C}$ temperature perturbation on a $1-\mathrm{km}$ standard fibre link, in open and closed loop

an amplitude modulation (AM) of the detected signal on the tilted photodiode. AM is directly converted into PM (Phase modulation) and detected as a phase perturbation term which is compensated by the phase compensator. Corrections of phase perturbations are corrupted by this parasitic phenomena and the performance of the system is then degraded. PDL is only related to the optical signal and independent of the modulation frequency. By upgrading the system to higher operation modulation frequencies, this problem could be reduced proportionally to the frequency.

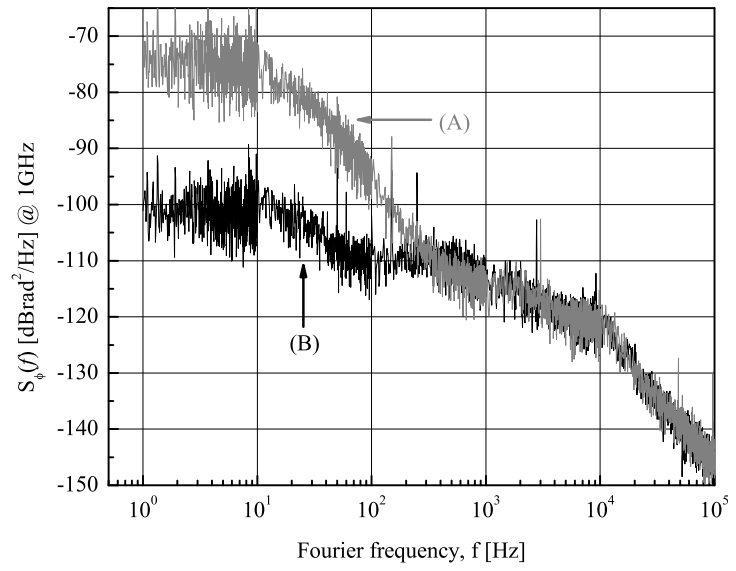

Figure 11: Measurements of the PZT phase noise reduction: Open loop phase noise measurement intentionally degraded (A) to show the rejection due to the PZT corrector (B)

\section{CHARACTERIZATION OF THE TWO 6 DIFFERENT SYSTEMS IN A FULL DIRECTIONAL LINK}

\section{A. Set up}

A full bidirectional compensated optical link $(2 \times 43$ $\mathrm{km}$ ) has been achieved by using the two fibres of the LNE-SYRTE to LPL link and by implementing the two previous compensation systems. This link is composed of various sections of buried optical cables of the France Telecom metropolitan network. The continuity of each optical fibre of $43 \mathrm{~km}$ is ensured by optical splicing and a global attenuation of $12 \mathrm{~dB}$ on each fibre is measured.

The low phase noise $100-\mathrm{MHz}$ local oscillator of LNE-SYRTE is transferred to LPL by using one of the two fibres of the link, and is phase compensated by the phase conjugator. At LPL, a signal phase coherent with the LNE-SYRTE local oscillator is detected and used as the input reference signal for the second optical link, connecting back LPL to LNE-SYRTE via the second $43-\mathrm{km}$ fibre. The optoelectronic system is installed on this link to compensate for the phase perturbations.

At LNE-SYRTE, we compare the phase detected signal coming from LPL with the local oscillator for characterizing the two dissemination systems. The use of two different systems allows systematic studies related to one of the two compensators, and thus allows to have a full characterization of the distribution system.

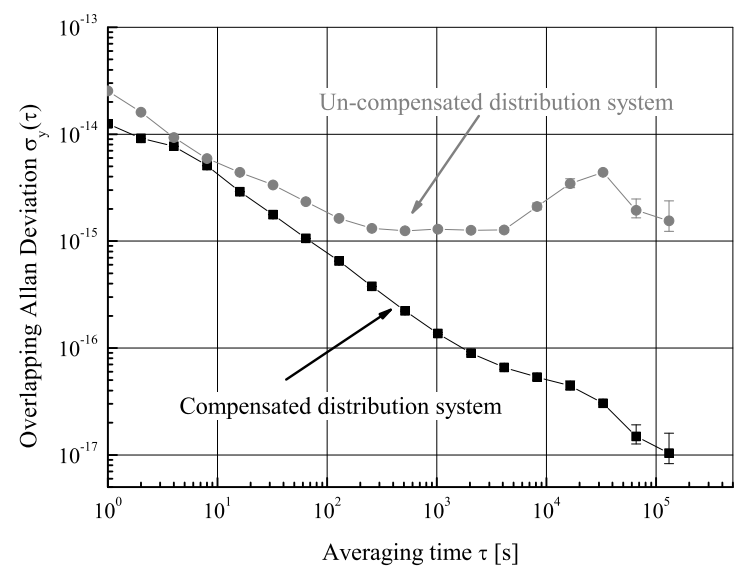

Figure 12: Frequency stability of the complete $86-\mathrm{km}$ distribution in open and closed loop

Figure 12 shows frequency stability measurements of the full bidirectional link $(2 \times 43 \mathrm{~km})$ between LNESYRTE and LPL. A fractional frequency stability of $10^{-17}$ at one day and $1.2 \times 10^{-14}$ at one second averaging time is obtained. These values of ADEV are calculated from the phase data measured on the link and filtered with a low-pass filter of $3 \mathrm{~Hz}$. 


\section{B. Direct application of the LNE-SYRTE to LPL} optical link

This link has been used to compare an optical frequency standard against an ultra-stable microwave oscillator, separated by a $43 \mathrm{~km}$ fibre link.

The optical standard is a $\mathrm{CO}_{2}$ laser stabilized on an $\mathrm{OsO}_{4}$ molecular absorption operating in the infrared domain, at $30 \mathrm{THz}$ [23]. At LNE-SYRTE, a 100-MHz signal is synthezised from the signal of a Cryogenic Sapphire Oscillator at $12 \mathrm{GHz}$, weakly phase locked on the signal of an H-MASER [24].

A femtosecond laser frequency comb allows the optical to microwave comparison and we demonstrate a resolution of $3 \times 10^{-14}$ at one second integration time $[25]$.

\section{Systems limitations}

As shown in figure 12, the frequency stability in closed loop is better than the open loop for all integration times, between $1 \mathrm{~s}$ and 1 day.

The short-term stability is mainly limited by the SNR at the detection, degraded by three contributions: the intrinsic noise of the distribution system, the optical attenuation in the fibre and the stimulated Brillouin scattering (SBS). Optical losses can be overcome by injecting powerful signals in the fibre, up to the Brillouin generation in order to avoid any laser source depletion. The direct amplitude modulation of the DFB bias current induces a broadening of the optical spectrum with a distribution of the energy within this spectrum and thus allows increasing the injected power level. The SBS leads also to a scattered wave detected as a white phase noise excess around the RF carrier with a few tens $\mathrm{MHz}$ width. This can be easily reduced by additional fibre Bragg grating filters (FBG), used in detection. The optoelectronic phase compensator, operating with two different modulation frequencies, separated by more than the Brillouin bandwidth, is less sensitive to this phenomenon.

In spite of its complexity, the phase conjugator presents a better phase noise ensuring thus a better short-term performance. The noise of the optoelectronic system is mainly degraded by additive amplitude noise, generated by the PZT corrector. One solution for improving the phase noise of each dissemination system is then to move to a higher operation RF frequency as $1 \mathrm{GHz}$. The long-term frequency stability is mainly limited by the phase conjugator which presents a rejection factor of only a few hundreds. Two main phenomena degrade the performance of the phase conjugator. Coherent leakage signals of $90 \mathrm{MHz}$ and $110 \mathrm{MHz}$ may induce parasitic phase shifts. The second limitation comes from optical feedback due to reflections of optical connectors or optical splicing along the link. A parasitic signal with an undetermined phase, varying in time, is thus detected and may be non-negligible compared to the main detected signal. At the detection, the ratio between the main signal and the parasitic signal is proportional to:

$$
\frac{10^{-\alpha\left(L-2 L_{R}\right)}}{R}
$$

where $\alpha$ is the optical attenuation in the fibre $[\mathrm{dB} / \mathrm{km}]$, $R$ is the power reflection coefficient, and $L_{R}[\mathrm{~km}]$ and $L[\mathrm{~km}]$ are respectively the reflection distance and the link lengh.

To ensure a sufficient compensation of the phase fluctuations introduced by the link, all parasitic noises should be $60 \mathrm{~dB}$ under the the detected signal. Such level could be reached by shifting the modulation frequency of the backward signal or by adding optical filters.

Finally, we are also confronted by a polarization effect, PMD (Polarization Mode Dispersion), which are detected as a chromatic dispersion with a random coefficient, leading to a random propagation delay on each way of the dissemination. Due to PMD, the principle of the compensation, based on the measurement of twice the perturbation after one round trip is not valid any more. One solution is the polarization scrambling of the injected optical signal, faster than the loop bandwidth.

\section{CONCLUSION \& PERSPECTIVES}

We have demonstrated for the first time the longdistance transfer of both short-term and long-term frequency stability of frequency standards, with low phase noise via telecom optical fibres. A stability of a few $10^{-14}$ at one second and $10^{-17}$ for one day integration has been obtained on an optical link of $86 \mathrm{~km}$.

In this way, we are able to compare two distant frequency standards operating in the microwave domain (CSO) and in the infrared domain (stabilized $\mathrm{CO}_{2}$ laser) with a resolution of $3 \times 10^{-14}$ at $1 \mathrm{~s}$.

The electronic phase conjugator presents the advantage to have an infinite dynamic allowing compensation of all phase perturbations, with a bandwidth limited by the round trip delay in the optical link. With a better phase noise, this setup shows a good rejection factor for the short-term but is limited to a few hundreds for the long-term. Even if, the user end of the dissemination system is really simple, the system remains complex. In contrast, despite its relative simplicity, the optoelectronic compensator shows a limited short-term rejection factor due to PMD and PDL, but a better long-term rejection, than the one achieved with the electronic compensation setup.

We intend to improve these results by one order of magnitude by upgrading both dissemination setups. Moving to a modulation frequency of $1 \mathrm{GHz}$ should improve the intrinsic noise of each system. Additional polarization scramblers should help to reduce polarization effects and amplitude noise caused by the PZT corrector. We also plan to modify the phase conjugator by frequency shifting the backward signal to suppress the effect of the optical feed-back and high-order products in frequency mixers, and to use of optical filters at the same time. 


\section{ACKNOWLEDGMENTS}

This work was supported by the European Space Agency / ESOC.

Thanks to Observatoire de Paris, the Laboratoire National de métrologie et d'Essais (LNE), Paris 13 Uni- versity, the French Research Ministry, CNRS, an 8 the Laboratoire d'Optronique, GIS FOTON, ENSSAT Lannion.

The authors thank D. Chambon and L. Volodimer. List of References
[1] P. Wolf, S. Bize, A. Clairon, A. N. Luiten, G. Santarelli and M. E. Tobar, Physics Review Letter, 90, 060402, 2003

[2] S. Bize et al., J. Phys. B, Atom. Mol. Opt. Phys. 38, pp. 449-468, 2005

[3] M. Fischer et al., Phys. Rev. Lett. 92, 230802, 2004

[4] E. Peik et al., Phys. Rev. Lett. 93, 170801, 2004

[5] J. Frisch, D. Bernstein, D. Brown and E. Cisneros, in IEEE Particle Accelerator Conference, 2002

[6] B. Shillue, ALMA Memo 443, 2002 (website: http: / www.alma.nrao.edu/memos/)

[7] K.H. Sato et al, in IEEE Trans. Instrum. Meas., vol. 41, pp. 385-389, 1992.

[8] R.T. Logan, G.F. Lutes, in Proc. of IEEE Frequency Control Symposium, 1992, p. 310-316.

[9] M. Calhoun, R. Wang, A. Kirk, W. Diener, G.J. Dick and R.L. Tjoelker, in Proc. of the $32^{\text {nd }}$ Annual Precise Time and Time Interval Systems and Applications Meeting, 2000, pp. 331-340.

[10] S. Bize et al, in C.R. Physique, vol. 5, 829, 2004

[11] I. Courtillot, A. Quessada, R. P. Kovacich, A. Brusch, D. Kolker, J-J. Zondy, G. D. Rovera and P. Lemonde et al, in Physical Review A, vol. 68, pp. 030501, 2003

[12] F. Riehle et al, in Appl. Phys. B, vol. 648, pp. 229-244, 2004

[13] U. Tanaka et al, in J. Phys. B, Atom. Mol. Opt. Phys. 36, pp. 545-551, 2003
[14] H. S. Margolis, G. P. Barwood, G. Huang, H. A. Klein, S. N. Lea, K. Szymaniec and P. Gill, in Science, vol. 306, pp. 1355-1358, 2004

[15] Th. Udem et al, in Physical Review Letters, vol. 86, 2001

[16] L.S. Ma et al, in Science, vol. 303, pp. 1843, 2004

[17] A. Amy-Klein et al, in Appl. Phys. B, vol. 78, pp. 2530,2004

[18] A. Bauch et al, in Metrologia, vol. 43, pp. 109-120, 2006

[19] J. Ye et al, in J. Opt. Soc. Am. B, vol. 20, pp. 1459, 2003

[20] K. W. Holman, D. J. Jones, D. D. Hudson and J. Ye, in Optics letters, vol. 29, 2004.

[21] K. Sato, T. Hara, S. Kuji, K. Asari, M. Nishio and N. Kawano, in IEEE Transactions on Instrumentation and Measurement, vol. 49, pp. 19-24, 2000.

[22] M. Calhoun, R. Sydnor and W. Diener, in The Interplanetary Network Progress Report, IPN PR 42-148, pp. 1-11, 2001.

[23] V. Bernard et al, in IEEE J. Quantum Electron, vol. 33, pp. 1282, 1997

[24] D. Chambon et al, in Review of Scientific Instruments, vol. $76,094704,2005$

[25] C. Daussy et al, in PRL, vol. 94, 203904, 2005 